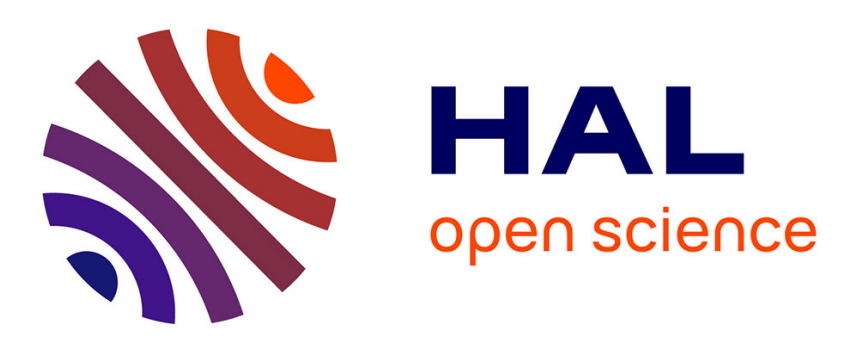

\title{
Can HIV epidemics among men who have sex with men be eliminated through participation in PrEP rollouts?
}

Sofía Jijón, Jean-Michel Molina, Dominique Costagliola, Virginie Supervie, Romulus Breban

\section{- To cite this version:}

Sofía Jijón, Jean-Michel Molina, Dominique Costagliola, Virginie Supervie, Romulus Breban. Can HIV epidemics among men who have sex with men be eliminated through participation in PrEP rollouts?. AIDS. Official journal of the international AIDS Society, 2021, 35 (14), pp.2347-2354. 10.1097/QAD.0000000000003012 . hal-03287483

\section{HAL Id: hal-03287483 \\ https://hal.science/hal-03287483}

Submitted on 15 Jul 2021

HAL is a multi-disciplinary open access archive for the deposit and dissemination of scientific research documents, whether they are published or not. The documents may come from teaching and research institutions in France or abroad, or from public or private research centers.
L'archive ouverte pluridisciplinaire HAL, est destinée au dépôt et à la diffusion de documents scientifiques de niveau recherche, publiés ou non, émanant des établissements d'enseignement et de recherche français ou étrangers, des laboratoires publics ou privés. 
AIDS, Publish Ahead of Print

DOI: 10.1097/QAD.0000000000003012

\section{Can HIV epidemics among men who have sex with men be eliminated through participation in PrEP rollouts?}

Sofía JIJÓN ${ }^{1}$, Jean-Michel MOLINA ${ }^{2}$, Dominique COSTAGLIOLA ${ }^{1}$, Virginie SUPERVIE ${ }^{1}$ and Romulus BREBAN ${ }^{3, \S}$

${ }^{1}$ Sorbonne Université, INSERM, Institut Pierre Louis d'épidémiologie et de Santé Publique (IPLESP UMR-S 1136), 75012 Paris, France

2 Département de Maladies Infectieuses, APHP-Hôpital Saint Louis, UMR 941 Inserm et Sorbonne Paris Cité, Paris, France

3 Institut Pasteur, Unité d’Épidémiologie des Maladies Émergentes, 75015 Paris, France

${ }^{\S}$ Corresponding author: Romulus Breban

Institut Pasteur, Unité d’Épidémiologie des Maladies Émergentes

25 rue du Docteur Roux

75015 Paris, France

+33(0)140613965

romulus.breban@pasteur.fr

E-mail addresses of authors:

SJ: sofia.jijon@iplesp.upmc.fr

J-MM: jean-michel.molina@aphp.fr

DC: dominique.costagliola@iplesp.upmc.fr

VS: virginie.supervie@inserm.fr

RB: romulus.breban@pasteur.fr 


\begin{abstract}
Objectives: To study the conditions under which PrEP coverage can eliminate HIV among men who have sex with men (MSM) in the Paris region.
\end{abstract}

Design: Mathematical modeling.

Methods: We propose an innovative approach, combining a transmission model with a game-theoretic model, for decision-making about PrEP use. Individuals at high risk of HIV infection decide to use PrEP, depending on their perceived risk of infection and the relative cost of using PrEP versus antiretroviral treatment (ART), which includes monetary and/or non-monetary aspects, such as price and access model of PrEP, consequences of being infected and lifelong ART.

Results: If individuals assessed correctly their infection risk, and the cost of using PrEP were sufficiently low, then the PrEP rollout could lead to elimination. Specifically, assuming 86\% PrEP effectiveness, as observed in two clinical trials, a minimum PrEP coverage of 55\% (95\% CI:43\%-64\%) among high-risk MSM would achieve elimination in the Paris region. A complete condom drop by MSM using PrEP slightly increases the minimum PrEP coverage required for elimination, by $\sim 1 \%$, while underestimation of their own HIV infection risk would require PrEP programs reduce the cost of using PrEP by a factor 2 to achieve elimination.

Conclusions: Elimination conditions are not yet met in the Paris region, where at most $47 \%$ of high-risk MSM were using PrEP as of mid-2019. Further lowering the cost of PrEP and promoting a fair perception of HIV risk are required and should be maintained in the long run, to maintain elimination status.

Keywords: Pre-exposure prophylaxis; HIV; men who have sex with men; behavioral epidemiology; game theory; prevention coverage

\title{
Introduction
}

In many settings, men who have sex with men (MSM) are most affected by HIV [1]. Preexposure prophylaxis (PrEP) is a highly effective prevention method recommended by the WHO for individuals at high risk of infection with HIV [2]. Both IPERGAY and PROUD clinical trials showed that PrEP can reduce HIV incidence among MSM by 86\% [3,4]. Modeling studies, elaborating on these results, suggested that PrEP has the potential to curtail, and even eliminate HIV epidemics, notably among MSM [5-8]. For instance, in the Netherlands, elimination would require 82\% PrEP coverage in the highest-risk group [6]. 
The question of whether it is possible to achieve a certain PrEP coverage in a population has not been addressed; modeling studies only assume that the coverage reaches certain values, which may not be granted in public health practice. It is therefore unclear whether, and under what conditions, target PrEP coverage levels, required to eliminate HIV epidemics, can be reached voluntarily and maintained in the long run. Currently, PrEP remains underutilized in many settings [9]. For instance, in the United States, 220,000-225,000 individuals were on PrEP as of April 2020 [10], still short of the CDC estimate that 1.2 million persons have indications for considering PrEP use [11]. Furthermore, a recent study shows that only two in five individuals keep using PrEP for $>2$ years [12].

Mathematical tools for modeling individual-level decision-making are offered by game theory [13-15]. We propose an innovative approach, combining an epidemic model at the population level, and a game-theoretic model for decision-making about PrEP at the individual level. We model PrEP adoption in a population at high risk of HIV infection, to determine whether and under what conditions certain PrEP coverage levels can be reached voluntarily. Particularly, we study the potential impact of PrEP among MSM in the Paris region of France, where universal antiretroviral treatment (ART) is in place, and PrEP is available for eligible individuals.

\section{Methods}

We built an HIV epidemic model (Figures S1 and S2, http://links.lww.com/QAD/C233) to describe the epidemiological context of an MSM community where eligible individuals make informed decisions about adopting PrEP. The decision making is modeled as a noncooperative game, where individuals act to maximize the utility of adopting PrEP, or, in other words, minimize the cost of using PrEP to avoid acquiring HIV and taking lifelong ART. Individual's decision is, however, indirectly influenced by that of others. The sum of all individuals' decisions determines the PrEP coverage, which, consequently, affects epidemic progression and the risk of acquiring HIV. The decision-making game model is thus intertwined with the epidemic model. Below, we describe the main features of our twocomponent model; see the Supplementary Material, http://links.lww.com/QAD/C233 (SM) for further details.

\section{The epidemic model}

The epidemic model stratifies the MSM population into two risk groups: (low and high), to account for heterogeneity in the infection risk. The majority of partnerships occur within the same risk group (i.e., assortative mixing) and individuals at high risk of infection drive the epidemic. The model also stratifies over HIV status, disease progression, diagnosis and the use of PrEP or ART. Once diagnosed, individuals immediately begin ART [16], no longer transmitting HIV. We varied PrEP effectiveness, denoted $\varepsilon$, from 0 to $100 \%$ to study suboptimal PrEP use. The PrEP coverage, $p$, was not fixed; rather, it was obtained through the decision-making game model (see below). 
We computed the effective reproduction number for the epidemic model, $R$, defined as the expected number of secondary cases caused by one infected individual, during his entire infectious period, in an uninfected population subject to control interventions [17,18]. PrEP use may change individuals' preference for other prevention tools, turning $R$ into a function of PrEP parameters. $R(p, \varepsilon)>1$ indicates epidemic persistence, meaning that an endemic state will be reached. Elimination requires $R(p, \varepsilon)<1$, such that the disease-free state will be reached. Elimination implies that incidence is reduced to zero in the studied population, but HIV can re-emerge in absence of control interventions, as it does not imply eradication. We say the epidemic is controlled using $\operatorname{PrEP}$ if $R(p, \varepsilon)$ decreases with the PrEP parameters, although the decrease is not below 1 . Our model shows that epidemic control and elimination can occur through PrEP, provided that two thresholds in PrEP effectiveness are exceeded; $\varepsilon \geq$ $\varepsilon_{\mathrm{C}}$ is required for epidemic control and $\varepsilon \geq \varepsilon_{\mathrm{E}}$ for epidemic elimination (see SM section 1.2.3, http://links.lww.com/QAD/C233). These thresholds are called the epidemic control and the epidemic elimination thresholds, respectively.

\section{The decision-making game model}

During an epidemic, individuals may adopt PrEP according to their perceived HIV risk [19], their perceived advantages and disadvantages of PrEP uptake, which includes quality of sex life, price [20] and access model of PrEP [21], adverse effects [22], social stigma [23], perceived consequences of being infected, which includes potential HIV-related stigma, regular clinical visits, lifelong ART, and other pros and cons. These factors, summarizing monetary and/or non-monetary aspects, are expressed in our decision-making model as costs perceived by the individual.

We assume that all high-risk MSM choose between two mutually-exclusive strategies. If an MSM decides not to use PrEP, then in the case of acquiring HIV he will start ART upon positive HIV diagnosis, and pay the cost of being infected and taking ART, called the cost of ART for simplicity, for the rest of his life; we use the notation $C_{\text {No-PrEP }}$ for the lifetime cost of this strategy. Otherwise, the MSM decides to adopt PrEP prevention, including regular testing for HIV. Thus, he takes and pays the cost of PrEP and, in the case of acquiring HIV despite PrEP uptake, being diagnosed and starting ART, pays the cost of ART for the rest of his life. We use the notation $C_{\text {PrEP }}$ for the lifetime cost of the second strategy. The total cost depends explicitly on the yearly costs perceived for ART and PrEP, the PrEP parameters, and, implicitly, the yearly risk of acquiring HIV.

We introduce $r$, the cost perceived for the strategy of adopting PrEP versus the cost perceived for the strategy of not adopting PrEP, which we call, for simplicity, the relative cost of PrEP versus ART. Hence, the balance of cost, when the probability to adopt PrEP is $p$, is

$$
C(p, r) \quad p C_{\mathrm{PrEP}}(p, r) \quad(1 \quad p) C_{\mathrm{No}-\mathrm{PrEP}}(p, \quad),
$$

where all functions and parameters, other than $p$, are given in our mathematical modeling. The value of $p$ that minimizes $C(p, \varepsilon, r)$, denoted $\hat{p}(, r)$, estimates the probability that a 
typical high-risk individual adopts PrEP, and also represents the voluntary PrEP coverage among high-risk MSM. The solution of the game represents an endemic state where individuals make decisions to adopt PrEP in stationary epidemiological context. We thus assumed that, in the long run, individuals stand by their decisions about adopting PrEP and we used our model only for long-term predictions.

\section{Application to the HIV epidemic among MSM in the Paris region}

We calibrated the epidemic model to represent the epidemiological context before the introduction of PrEP [24,25], and obtained many HIV parameter sets, to reveal uncertainty in the model output (SM section 2 and Tables S1-S4, http://links.lww.com/QAD/C233). In our baseline scenario, we assumed that MSM on PrEP get tested for HIV quarterly, according to the French recommendations [26]. The testing frequency on PrEP was thus much higher than that observed off PrEP, as data shows $\sim 3$ years for the mean time from HIV infection to diagnosis among MSM before the introduction of PrEP (personal communication with VS). We further assumed that individuals have a fair perception of their infection risk when making decisions about PrEP use; the infection risk was determined by the force of HIV infection of the epidemic model. Furthermore, MSM were assumed to drop condom use from $30 \%$ to $20 \%$ when adopting PrEP [3], and the condom effectiveness was $58 \%-80 \%$ [27]. Sensitivity scenarios were explored assuming that i) MSM misperceived their risk of acquiring HIV, or ii) MSM adopting PrEP completely dropped condom use [28], (SM Section 3, http://links.lww.com/QAD/C233).

\section{Results}

About 500 parameter sets calibrated our epidemic model to the HIV epidemiology among MSM in the Paris region, before the introduction of PrEP: total yearly mean incidence was $1.3 \%$, prevalence was $17 \%$, and $17 \%$ of the MSM living with HIV were undiagnosed (Table S3, http://links.lww.com/QAD/C233). The mean number of MSM was $~ 111,000$, of which $13 \%$ (i.e., 14,200 ) were at high risk of infection and eligible for PrEP. Yearly incidence for high-risk MSM was $7 \%$. The model parameters implied that the PrEP rollout had two effects: first, it offered the prevention benefits of the regimen, and, second, it behaved as a test-andtreat strategy [29,30], imposing a major change in HIV testing practice (SM Section 3.1 and Figure S3, http://links.lww.com/QAD/C233).

\section{The voluntary PrEP coverage if individuals perceived correctly HIV infection risk}

We first investigated a typical parameter set calibrating our model; Table S2, http://links.lww.com/QAD/C233. The PrEP coverage starts at zero, before introducing PrEP, and then, in the long term, reaches an equilibrium value where the expected cost of adopting PrEP is minimum. The final value reached depends on HIV parameters of the epidemic before the introduction of PrEP, the PrEP effectiveness, $\varepsilon$, and the perceived relative cost of PrEP versus ART, $r$. Figure 1A shows the voluntary PrEP coverage reached among high-risk 
MSM, $\hat{p}(, r)$. Figure 1B shows the corresponding relative reduction in HIV incidence in the MSM community. Each of these two figures shows three regions:

- Region III, where no high-risk MSM adopts PrEP, because the perceived relative cost of PrEP versus ART is too high. Therefore, HIV remains endemic, unaffected by the introduction of PrEP (i.e., no reduction in incidence);

- Region II, where some, but not enough, high-risk MSM adopt PrEP, since the relative cost remains high. The epidemic is controlled and incidence decreases, but not enough for elimination (i.e., $R(\hat{p}, \varepsilon)>1$ );

- Region I, where PrEP is offered at low relative cost. This allows reaching high levels of PrEP coverage ( 54-75\%) and the epidemic can be eliminated; for Region I, $R(\hat{p}$, $\varepsilon)<1$. HIV elimination for low PrEP effectiveness (bottom part of Figure 1A) occurs as a consequence of the test-and-treat effect of the PrEP rollout; consequently, $\varepsilon_{\mathrm{C}}=\varepsilon_{\mathrm{E}}$ $=0 \%$. In this case, MSM taking PrEP are poorly protected against HIV. However, they are diagnosed and treated very early in the course of infection, because they get tested for HIV every three months. Early diagnosis and treatment prevent further HIV transmission. In contrast, when PrEP effectiveness is high, most on-PrEP MSM do not acquire HIV, so the test-and-treat benefit of the PrEP rollout is marginal. It is PrEP, particularly its high effectiveness, that contributes decisively to epidemic elimination. If $\varepsilon=86 \%$, as observed in the IPERGAY and PROUD trials, a minimum PrEP coverage of $56 \%$ should be reached among high-risk MSM, to eliminate HIV; Figure S4, http://links.lww.com/QAD/C233.

It is important to note that elimination is temporary, as the disease-free state is unstable. Indeed, once the epidemic is eliminated, individuals perceive HIV risk as being low and may reevaluate the pros and cons of PrEP. In turn, this may severely increase the relative cost of PrEP versus ART, since the epidemic is considered to be eliminated and prevention is perceived as no longer needed. As fewer individuals consider PrEP use, the PrEP coverage decreases and the HIV epidemic dynamics in Region I can enter Region II, where the epidemic reemerges and becomes again of public health concern.

We generated the outputs in Figure 1 using each of the $~ 500$ parameter sets obtained through calibration, to estimate uncertainty intervals for our results (SM Section 2, http://links.lww.com/QAD/C233). Figure 2A shows the probability that HIV is eliminated, as a function of $\varepsilon$ and $r$. The probability is high on the left, where Region $\mathrm{I}$ is found, and declines severely toward Region II. In Figure 2B, we illustrate the boundaries between Regions I and II (continuous line), and between Regions II and III (dashed line); the threeregion structure appears robust to parameter uncertainties. Additionally, when $\varepsilon=86 \%$, we found that the $95 \%$ confidence interval (CI) of the minimum PrEP coverage needed for elimination (i.e., $55 \%$ ) is $43 \%-64 \%$. 


\section{Sensitivity scenarios}

We assumed that individuals could misperceive their HIV risk when deciding to adopt PrEP, and repeated our analyses. Specifically, rather than having a fair sense of HIV risk, based on the force of infection, high-risk MSM could get a sense of HIV risk from, for instance, the proportion of their high-risk MSM peers being diagnosed each year with HIV (SM Section 3.3.1, http://links.lww.com/QAD/C233), assuming full disclosure from HIV-diagnosed MSM. The voluntary PrEP coverage computed for this scenario is illustrated in Figure 3 and reveals a qualitatively similar structure to that in Figure 1. However, when high-risk MSM misinterpret and underestimate their HIV risk, Region I is smaller, implying that the relative cost of PrEP versus ART must be lower to achieve epidemic elimination. In particular, when $\varepsilon=86 \%$, the relative cost needed for epidemic elimination decreases by a factor of $\sim 2$, making Region I harder to reach in practice of public health.

We performed another sensitivity analysis, where we analyzed PrEP-driven condom drop. In our baseline scenario, MSM dropped condom use from 30\% to 20\% when adopting PrEP. Similar results were obtained assuming that PrEP users stopped using condoms completely (SM Section 3.3.2 and Figure S5, http://links.lww.com/QAD/C233). We thus concluded that condom drop is not a major factor against HIV elimination when PrEP effectiveness is high. Specifically, epidemic elimination where $\varepsilon=86 \%$ requires a coverage of $>57 \%$, rather than $>56 \%$ in the baseline scenario.

\section{Perspectives on the PrEP rollout in the Paris region}

In 2016, a PrEP rollout started in the Paris region, offering fully subsidized PrEP to eligible individuals. As mentioned before, under the baseline scenario, for 86\% PrEP effectiveness, we found that at least 55\% (95\%CI: 43\%-64\%) of the high-risk MSM would need to take PrEP for the HIV epidemic be eliminated. Since, according to our calibration, the estimated number of PrEP-eligible MSM in the Paris region is 14,200 (95\%CI: 9,200-23,000), this means that 7,700 (95\%CI: 5,800-10,100) high-risk MSM should remain on PrEP for the long term. This is an objective to be reached. As of mid-2019, 6,700 men were on PrEP in the Paris region [31], with a marked growing trend. However, the 30-month dropout rate was $\sim 32 \%$ [32]. The PrEP coverage among high-risk MSM was then estimated to be at most 47\% (95\%CI: 30\%-73\%), assuming that all men on PrEP were indeed high-risk MSM, which is probably an overestimation. If all these MSM remained on PrEP for the long term, our model predicted epidemic control (i.e., Region II), with a reduction of 90\% (95\%CI: 81\%-100\%) in HIV incidence at the new endemic state.

\section{Discussion}

We addressed the role of individual-level decision-making in the potential impact of PrEP on the HIV epidemic, identified the conditions for epidemic control or elimination, and estimated PrEP coverage levels which may be reached voluntarily. We obtained four major findings for PrEP rollouts. First, HIV epidemics can be eliminated provided that the relative 
cost of using PrEP versus ART is sufficiently low. Second, frequent HIV testing while taking PrEP can compensate for poor PrEP adherence and act as a test-and-treat intervention. Third, HIV risk perception may play a major role for elimination, while drop in condom use among PrEP users may not. Fourth, epidemic elimination may be only temporary.

We applied our model to the Paris region. Assuming a PrEP effectiveness of 86\%, as reported in two major clinical trials, we found that at least 55\% (95\%CI: 43\%-64\%) of the high-risk MSM would need to be on PrEP to achieve HIV elimination. As of mid-2019, at most $47 \%$ high-risk MSM were on PrEP in the Paris region, meaning that the PrEP-rollout protocol did not reduce enough the cost of PrEP for epidemic elimination, so far. Still, a recent update on new HIV diagnoses in Paris [33] shows that the numbers among French-born MSM decreased by 28\%, between 2015 and 2018, with no significant decrease for other MSM. This decrease could be partly due to the PrEP rollout starting in 2016, and, according to our modeling, should continue in the near future. In two other settings, a moderate-high PrEP coverage has been quickly reached. The region of New South Wales witnessed a rapid PrEP rollout ( 9,000 MSM on PrEP within 2 years) during an implementation study providing PrEP for free at several sites, including public HIV and sexual health services, and private general practices with expertise in ART prescription [34]. About $41 \%$ of the high-risk MSM in Australia were on PrEP in 2017 [35]. Since April 2018, PrEP is subsidized by the Australian government and can be prescribed by any practitioner [36]. In San Francisco, a citywide-coordinated PrEP rollout, within the Getting to Zero program, strongly promoted PrEP and offered PrEP for free or at low monetary cost, through insurance benefits or patient assistance programs. Close to 50\% of the eligible MSM were on PrEP in 2017 in San Francisco [37]. Although these levels of PrEP coverage contributed to decreasing HIV transmission $[34,37,38]$, HIV elimination has not been reported.

Moving toward epidemic elimination will require further decreasing the cost perceived for PrEP uptake, which involve reducing monetary and non-monetary barriers to PrEP uptake, such as difficulties in accessing PrEP, pill burden, tolerability of the molecules, social stigma and discrimination, and the acquisition of other sexually transmitted infections in case of dropping condom use [21-23]. Online tools [39], home-based programs [40], long-lasting injectable versions of PrEP [41], rather than daily or on-demand pills, allowing trained general practitioners to prescribe PrEP and interventions that increase awareness, motivations and behavioral skills about risk reduction [42] may also help reduce the perceived cost of PrEP and decrease the drop-out rate. If feasible, estimating the cost of PrEP relative to that of ART would make it possible to predict the resulting PrEP coverage, depending on the PrEP rollout. However, in practice, it may be very complex to estimate this cost, as it depends on many factors. Nevertheless, it is very important to note that estimating the cost is not strictly needed. Indeed, interventions which intuitively increase the accessibility and affordability of PrEP, may be proposed and thus contribute to reducing the cost, placing the PrEP rollout in the right direction. Then, the reduction in cost can be indirectly appreciated by monitoring the increase in PrEP coverage and the decrease in HIV incidence, which can serve as indicators for how far the PrEP rollout is from achieving elimination. 
Moving toward epidemic elimination will also require reaching MSM who may not perceive themselves at high risk, and thus require a lower cost for adopting PrEP, in order to join the prevention effort. Recent studies found that high-risk individuals can underestimate their HIV risk [43] and there are many missed opportunities for PrEP uptake [44]. Specifically, in France, $>90 \%$ of the recently infected individuals were eligible for PrEP [44]. Therefore, assessing and communicating individual-level risk for acquiring HIV remains a key objective for achieving elimination. Promoting a fair perception of HIV risk can be achieved through, not only advertising and marketing PrEP [45], but also through using electronic health records to identify high-risk MSM [46].

Importantly, if HIV is eliminated, interventions will be needed so individuals keep perceiving a low cost for PrEP and fair perception of HIV risk, to maintain a high PrEP coverage. Otherwise, HIV can reemerge and reach again an endemic state of concern for public health. The situation is similar to that of vaccination prevention, which requires continuous vaccine coverage even though the disease is declared to be eliminated [47].

Our study has some notable limitations. First, some of our modeling assumptions may be applicable only to the Parisian setting and other urban high-income settings. Second, we assumed that individuals act out of self-interest and do not cooperate to avoid getting infected by HIV. Modeling PrEP adoption through other theories of health behavior, considering for instance interactions between individuals [15], remains a subject to be studied in further work. Third, assuming full disclosure of HIV status in our sensitivity scenario may be unlikely. Also, we assumed that MSM are homogeneous regarding risk perception, while in reality, fair perception certainly co-exists with misperception. Fourth, we did not account for migration or travel [48], nor social or sex networks, due to lack of specific data, nor for condom drop among non-PrEP users [49], which could influence elimination efforts. Fifth, our estimates of the number of high-risk individuals, who should be on PrEP for HIV elimination, depend on the size of the MSM community, which is a metric difficult to estimate. Also, the number of high-risk MSM on PrEP currently reported, and hence the PrEP coverage, may represent an overestimate because establishing PrEP eligibility relies on selfreported behavior, which is difficult to appraise by practitioners.

\section{Conclusion}

Perception of the cost of PrEP and of HIV risk are two important levers to increase voluntary use of PrEP, reach coverage levels necessary to eliminate HIV, and maintain elimination in the context of less epidemic adversity. Current PrEP rollouts should aim at lowering the perceived cost of using PrEP and promoting a fair perception of the risk of acquiring HIV, to realize the full potential of PrEP prevention. 


\section{Authors' contributions}

SJ, VS an RB conceived the model. SJ conducted the numerical simulations. All authors participated to the writing of the manuscript, analysis and interpretation of the results. All authors read and approved the final manuscript.

\section{Acknowledgements}

SJ was supported by an ANRS fellowship and a PhD fellowship from the French Ministry of Higher Education and Research, obtained via the Public Health Doctoral Network coordinated by the EHESP. The sponsors had no role in the study.

\section{List of abbreviations}

ART: Antiretroviral treatment; MSM: Men who have sex with men; PrEP: Pre-exposure prophylaxis; CI: confidence interval.

\section{References}

1 Beyrer C, Baral SD, Collins C, Richardson ET, Sullivan PS, Sanchez J, et al. The global response to HIV in men who have sex with men. Lancet (London, England) 2016; 388:198-206.

2 World Health Organization. Policy brief: WHO expands recommendation on oral preexposure prophylaxis of HIV infection (PrEP). World Heal. Organ. 2015. Available at: https://apps.who.int/iris/handle/10665/197906. [Website accessed on April 20, 2021]

3 Molina J-M, Capitant C, Spire B, Pialoux G, Cotte L, Charreau I, et al. On-Demand Preexposure Prophylaxis in Men at High Risk for HIV-1 Infection. $N$ Engl $J$ Med 2015; 373:2237-2246.

4 McCormack S, Dunn DT, Desai M, Dolling DI, Gafos M, Gilson R, et al. Preexposure prophylaxis to prevent the acquisition of HIV-1 infection (PROUD): Effectiveness results from the pilot phase of a pragmatic open-label randomised trial. Lancet 2016; 387:53-60.

5 Jenness SM, Goodreau SM, Rosenberg E, Beylerian EN, Hoover KW, Smith DK, et al. Impact of the Centers for Disease Control's HIV Preexposure Prophylaxis Guidelines for Men Who Have Sex With Men in the United States. J Infect Dis 2016; 214 :1800-1807.

6 Rozhnova G, Heijne J, Bezemer D, van Sighem A, Presanis A, De Angelis D, et al. Elimination prospects of the Dutch HIV epidemic among men who have sex with 
men in the era of preexposure prophylaxis. Aids 2018; 32:2615-2623.

$7 \quad$ Scott N, Stoové M, Kelly SL, Wilson DP, Hellard ME. Achieving 90-90-90 Human Immunodeficiency Virus (HIV) Targets Will Not Be Enough to Achieve the HIV Incidence Reduction Target in Australia. Clin Infect Dis 2018; 66:1019-1023.

8 Hansson D, Stromdahl S, Leung KY, Britton T. Introducing pre-exposure prophylaxis to prevent HIV acquisition among men who have sex with men in Sweden: insights from a mathematical pair formation model. BMJ Open 2020; 10:e033852.

9 Cohen J. Concern as HIV prevention strategy languishes. Science 2018; 359:1205.

10 PrEP Watch. Global PrEP Tracker. 2010. Available at: https://www.prepwatch.org/resource/global-prep-tracker/. [Website accessed on April $20,2021]$.

11 Smith DK, Van Handel M, Wolitski RJ, Stryker JE, Hall HI, Prejean J, et al. Vital signs: Estimated percentages and numbers of adults with indications for preexposure prophylaxis to prevent HIV acquisition - United States, 2015. Morb Mortal Wkly Rep 2015; 64:1291-1295.

12 Coy KC, Hazen RJ, Kirkham HS, Delpino A, Siegler AJ. Persistence on HIV preexposure prophylaxis medication over a 2-year period among a national sample of 7148 PrEP users, United States, 2015 to 2017. J Int AIDS Soc 2019; 22:e25252.

13 Verelst F, Willem L, Beutels P. Behavioural change models for infectious disease transmission: a systematic review (2010-2015). J R Soc Interface 2016;

13:20160820.

14 Manfredi P, D’Onofrio A, editors. Modeling the Interplay Between Human Behavior and the Spread of Infectious Diseases. New York, NY: Springer New York; 2013.

15 Chang SL, Piraveenan M, Pattison P, Prokopenko M. Game theoretic modelling of infectious disease dynamics and intervention methods: a review. J Biol Dyn 2020; 14:57-89.

Dabis F. Achieving 90-90-90 - Implications for HIV epidemic control. 10th IAS Conference on HIV Science. Mexico city, Mexico : July 2019. Avaiable at: http://programme.ias2019.org/Programme/Session/4 [Website accessed on April 20, 2021].

17 Jacquez JA, Simon CP, Koopman J, Sattenspiel L, Perry T. Modeling and analyzing HIV transmission: the effect of contact patterns. Math Biosci 1988; 92:119-199. 
van den Driessche P, Watmough J. Reproduction numbers and sub-threshold endemic equilibria for compartmental models of disease transmission. Math Biosci 2002; 180:29-48.

Bull L, Dimitrijevic P, Beverley S, Scarborough A, Mandalia S, Dosekun O, et al. Perceived need of, and interest in, HIV pre-exposure prophylaxis amongst men who have sex with men attending three sexual health clinics in London, UK. Int $J$ STD AIDS 2018; 29:435-442.

van Dijk M, de Wit JBF, Guadamuz TE, Martinez JE, Jonas KJ. Slow uptake of PrEP: Behavioral predictors and the influence of price on PrEP uptake among MSM with a high interest in PrEP. AIDS Behav 2021.

21 Gilson RI, Clutterbuck DJ, Chen ZE. Demand for pre-exposure prophylaxis for HIV and the impact on clinical services: Scottish men who have sex with men perspectives. Int J STD AIDS 2018; 29:273-277.

22 Thomann M, Grosso A, Zapata R, Chiasson MA. 'WTF is PrEP?': attitudes towards pre-exposure prophylaxis among men who have sex with men and transgender women in New York City. Cult Health Sex 2017; 20:772-786.

23 Brooks RA, Nieto O, Landrian A, Donohoe TJ. Persistent stigmatizing and negative perceptions of pre-exposure prophylaxis (PrEP) users: implications for PrEP adoption among Latino men who have sex with men. AIDS Care 2019; 31:427-435.

24 Marty L, Cazein F, Panjo H, Pillonel J, Costagliola D, Supervie V. Revealing geographical and population heterogeneity in HIV incidence, undiagnosed HIV prevalence and time to diagnosis to improve prevention and care: estimates for France. J Int AIDS Soc 2018; 21:e25100.

25 Santé Publique France. Surveillance de l'infection à VIH (dépistage et déclaration obligatoire), 2010-2017. Availabe at: https://www.santepubliquefrance.fr/maladies-ettraumatismes/infections-sexuellement-transmissibles/vih-sida/documents/bulletinnational/bulletin-de-sante-publique-infection-a-vih.-mars-2019 [Website accessed on April 20, 2021].

26 CNS, ANRS. Prise en charge médicale des personnes vivant avec le VIH. 2018. Available at: https://cns.sante.fr/actualites/prise-en-charge-du-vih-recommandationsdu-groupe-dexperts/ [Website accessed on April 20, 2021]

27 Smith DK, Herbst JH, Zhang X, Rose CE. Condom Effectiveness for HIV Prevention by Consistency of Use Among Men Who Have Sex With Men in the United States. JAIDS J Acquir Immune Defic Syndr 2015; 68:337-344. 
changes in condom use and uptake of HIV pre-exposure prophylaxis by gay and bisexual men in Melbourne and Sydney, Australia: results of repeated behavioural surveillance in 2013-17. Lancet HIV 2018; 3018:1-9.

29 Kretzschmar ME, van der Loeff MF, Birrell PJ, De Angelis D, Coutinho RA. Prospects of elimination of HIV with test-and-treat strategy. Proc Natl Acad Sci $U$ S A 2013; 110:15538-15543.

30 WHO. Prevent HIV, test and treat all. 2016. Available at: https://www.who.int/hiv/pub/progressreports/2016-progress-report/en/ [Website accessed on April 20, 2021].

31 EPI-PHARE. Suivi de l'utilisation de Truvada ${ }^{\circledR}$ ou génériques pour une prophylaxie pré-exposition (PrEP) au VIH à partir des données du Système National des Données de Santé (SNDS). 2019. Available at: https://www.epi-phare.fr/rapports-detudes-etpublications/suivi-de-lutilisation-de-truvada-ou-generiques-pour-une-prophylaxiepre\%E2\%80\%90xposition-prep-au-vih/ [Website accessed on April 20, 2021]

32 Costagliola D, Ghosn J, Spire B et al. PrEP persistence and associated factors: an analysis from the ANRS Prevenir study. 17th European AIDS Conference, November 6-9, 2019, Basel. Abstract PS11/1.

33 Santé Publique France. Dépistage du VIH et découvertes de séropositivité VIH à Paris, données 2018. Available at: https://www.santepubliquefrance.fr/lesactualites/2019/depistage-du-vih-et-decouvertes-de-seropositivite-vih-a-paris-donnees2018 [Website accessed on April 20, 2021]

34 Grulich AE, Guy R, Amin J, Jin F, Selvey C, Holden J, et al. Population-level effectiveness of rapid, targeted, high-coverage roll-out of HIV pre-exposure prophylaxis in men who have sex with men: the EPIC-NSW prospective cohort study. Lancet HIV 2018; 3018:1-9.

35 Kirby Institute. HIV diagnoses in Australia drop to lowest number in 18 years. Available at: https://kirby.unsw.edu.au/news/hiv-diagnoses-australia-drop-lowestnumber-18-years [Website accessed on April 20, 2021]

36 Australasian Society for HIV VH and SHM (ASHM). HIV PrEP available on PBS in Australia from 1 April. 2018. Available at https://www.ashm.org.au/news/hiv-PrEPavailable-announced-on-pbs/ [Website accessed on April 20, 2021].

37 San Francisco Department of Public Health. HIV Epidemiology Annual Report 2017. Available at: https://www.sfdph.org/dph/comupg/oprograms/HIVepiSec/HIVepiSecReports.asp [Website accessed on June 20, 2020]. 
Smith DK, Sullivan PS, Cadwell B, Waller LA, Siddiqi A, Mera-Giler R, et al.

Evidence of an Association of Increases in Pre-exposure Prophylaxis Coverage

With Decreases in Human Immunodeficiency Virus Diagnosis Rates in the United States, 2012-2016. Clin Infect Dis 2020; 71: 3144-3151.

Getting to zero San Francisco. PrEP Committee. Available at:

http://www.gettingtozerosf.org/prep-committee/ [Website accessed on April 20, 2021]

Siegler AJ, Mayer KH, Liu AY, Patel RR, Ahlschlager LM, Kraft CS, et al.

Developing and Assessing the Feasibility of a Home-based Preexposure

Prophylaxis Monitoring and Support Program. Clin Infect Dis 2019; 68:501-504.

Marshall BDL, Goedel WC, King MRF, Singleton A, Durham DP, Chan PA, et al.

Potential effectiveness of long-acting injectable pre-exposure prophylaxis for HIV prevention in men who have sex with men: a modelling study. Lancet HIV 2018; 3018:1-8.

Dubov A, Altice FL, Fraenkel L. An Information-Motivation-Behavioral Skills Model of PrEP Uptake. AIDS Behav 2018; 22:3603-3616.

43 Blumenthal J, Jain S, Mulvihill E, Sun S, Hanashiro M, Ellorin E, et al. Perceived Versus Calculated HIV Risk. JAIDS J Acquir Immune Defic Syndr 2019; 80:e23e29.

Lions C, Cabras O, Cotte L, Huleux T, Gagneux-Brugnon A, Makinson A, et al. Missed opportunities of HIV pre-exposure prophylaxis in France: a retrospective analysis in the French DAT'AIDS cohort. BMC Infect Dis 2019; 19:278.

Amico KR, Bekker L. Global PrEP roll-out : recommendations for programmatic success. Lancet HIV 2019; 6:e137-e140.

Marcus JL, Hurley LB, Krakower DS, Alexeeff S, Silverberg MJ, Volk JE. Use of electronic health record data and machine learning to identify candidates for HIV pre-exposure prophylaxis: a modelling study. Lancet HIV 2019; 3018:1-8.

Jijón S, Supervie V, Breban R. Prevention of treatable infectious diseases: A gametheoretic approach. Vaccine 2017; 35:5339-5345.

Palk L, Gerstoft J, Obel N, Blower S. A modeling study of the Danish HIV epidemic in men who have sex with men: travel, pre-exposure prophylaxis and elimination. Sci Rep 2018; 8:16003. 
Figure 1. The voluntary PrEP coverage and its impact on HIV incidence, assuming fair risk perception

Color maps of (A) the voluntary PrEP coverage among high-risk men who have sex with men (MSM), $\hat{p}$, and (B) the corresponding reduction in the overall endemic HIV incidence rate, as functions of $\varepsilon$ and $r$, assuming that individuals have a fair perception of HIV risk. The model outputs were obtained for one typical parameter set calibrating our model. Three regions were identified, depending on $\hat{p}$ : Region III, where $r$ is high and no MSM uses PrEP ( $\hat{p}=0 \%$ ), so HIV incidence is not reduced; Region II, where some, but not enough MSM use PrEP, since $r$ remains high, and thus the epidemic is controlled; and Region I (marked by blue stripes), where epidemic elimination is possible.

A

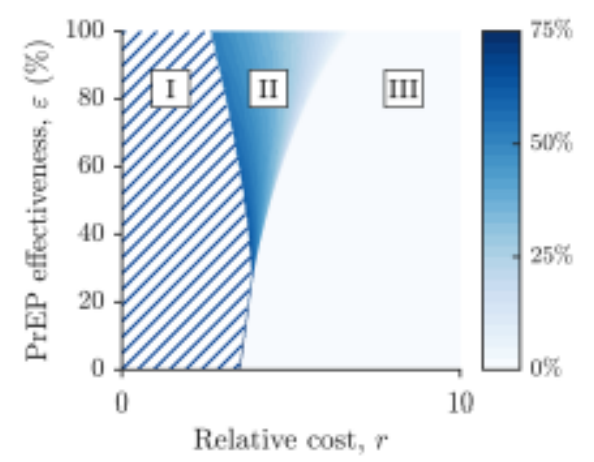

B

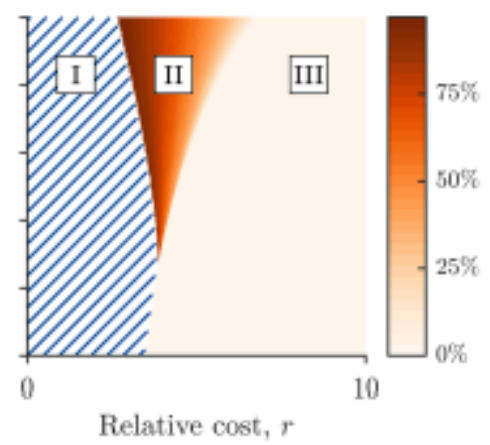

Figure 2. The probability of HIV elimination and boundary uncertainty for the threeregion structure

(A) The probability of HIV epidemic elimination due to voluntary PrEP coverage, obtained from the $\sim 500$ calibrated parameter sets. (B) The boundaries (the mean is represented as a line and the 95\% confidence interval as grey area) between Regions I and II (continuous line), and between Regions II and III (dashed line).

A

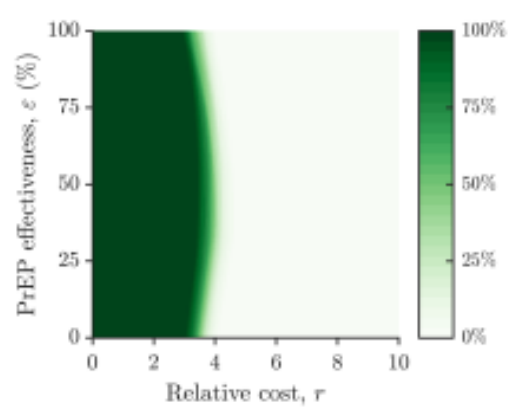

B

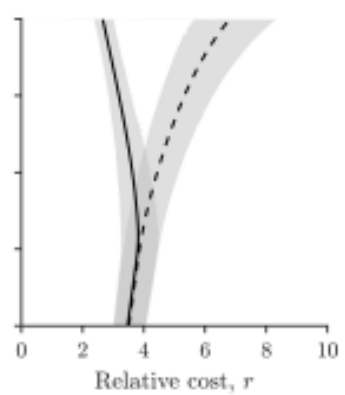


Figure 3. Sensitivity analyses for the baseline scenario

Decision-making based on misperceived risk of acquiring HIV can significantly reduce the size of Region I, where epidemic elimination is possible (blue stripes), despite high levels of PrEP effectiveness. Note that risk misperception also enlarges Region III, where no MSM is willing to adopt PrEP.
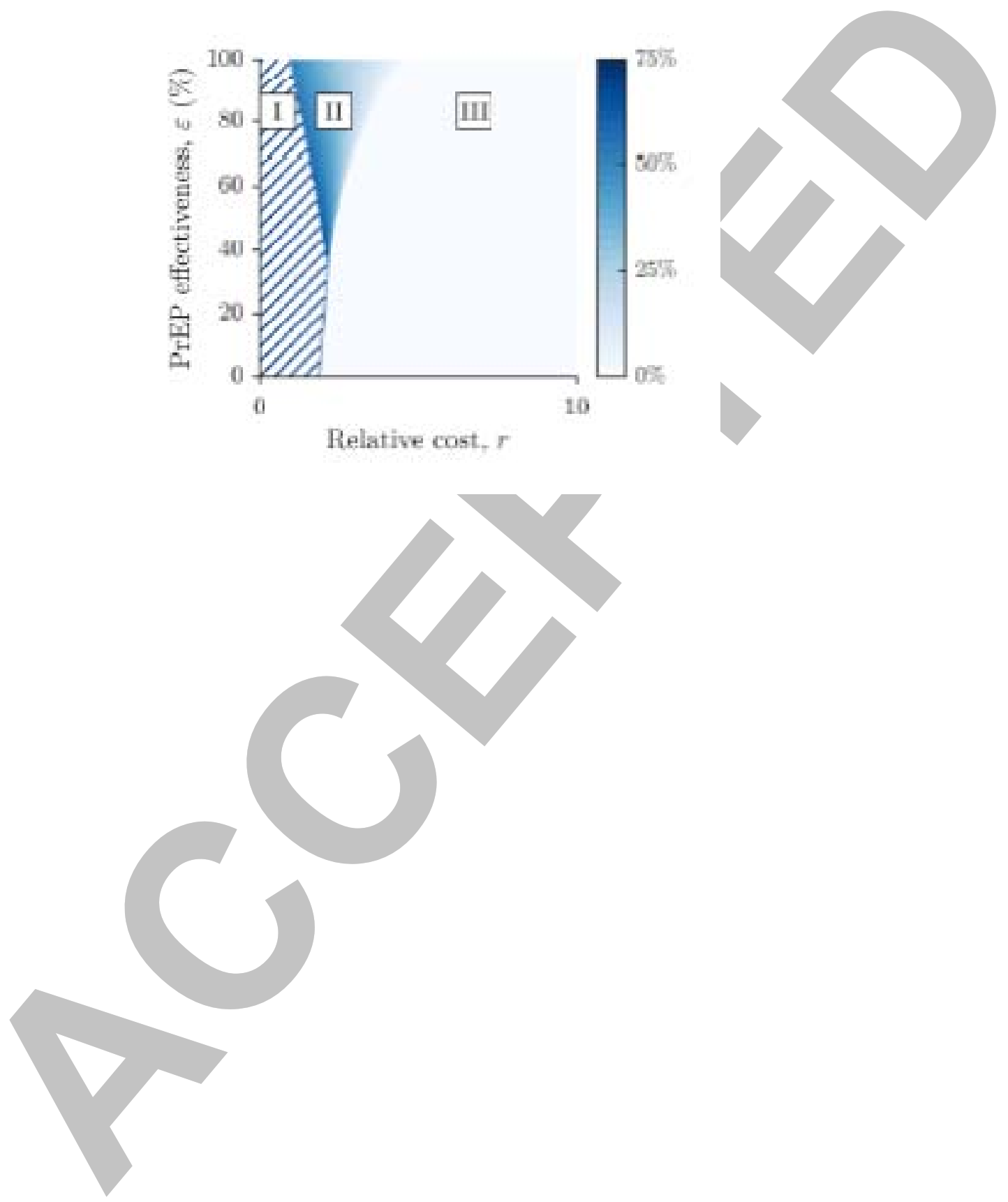

Copyright @ 2021 Wolters Kluwer Health, Inc. Unauthorized reproduction of this article is prohibited. 